\title{
Detection of diffuse TeV gamma-ray emission from the neaby starburst galaxy NGC 253
}

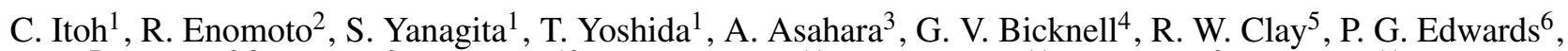
S. Gunji ${ }^{7}$, S. Hara ${ }^{3,8}$, T. Hara ${ }^{9}$, T. Hattori ${ }^{10}$, Shin. Hayashi ${ }^{11}$, Sei. Hayashi ${ }^{11}$, S. Kabuki ${ }^{2}$, F. Kajino ${ }^{11}$, H. Katagiri ${ }^{2}$, A. Kawachi ${ }^{2}$, T. Kifune ${ }^{12}$, H. Kubo ${ }^{3}$, J. Kushida ${ }^{3,8}$, Y. Matsubara ${ }^{13}$, Y. Mizumoto ${ }^{14}$, M. Mori ${ }^{2}$, H. Moro ${ }^{10}$, H. Muraishi ${ }^{15}$, Y. Muraki ${ }^{13}$, T. Naito ${ }^{9}$, T. Nakase ${ }^{10}$, D. Nishida ${ }^{3}$, K. Nishijima ${ }^{10}$, K. Okumura ${ }^{2}$, M. Ohishi $^{2}$, J. R. Patterson ${ }^{5}$, R. J. Protheroe ${ }^{5}$, K. Sakurazawa ${ }^{8}$, D. L. Swaby ${ }^{5}$, T. Tanimori ${ }^{3}$, F. Tokanai ${ }^{7}$, K. Tsuchiya ${ }^{2}$, H. Tsunoo ${ }^{2}$, T. Uchida ${ }^{2}$, A. Watanabe ${ }^{7}$, S. Watanabe ${ }^{3}$, and T. Yoshikoshi ${ }^{16}$

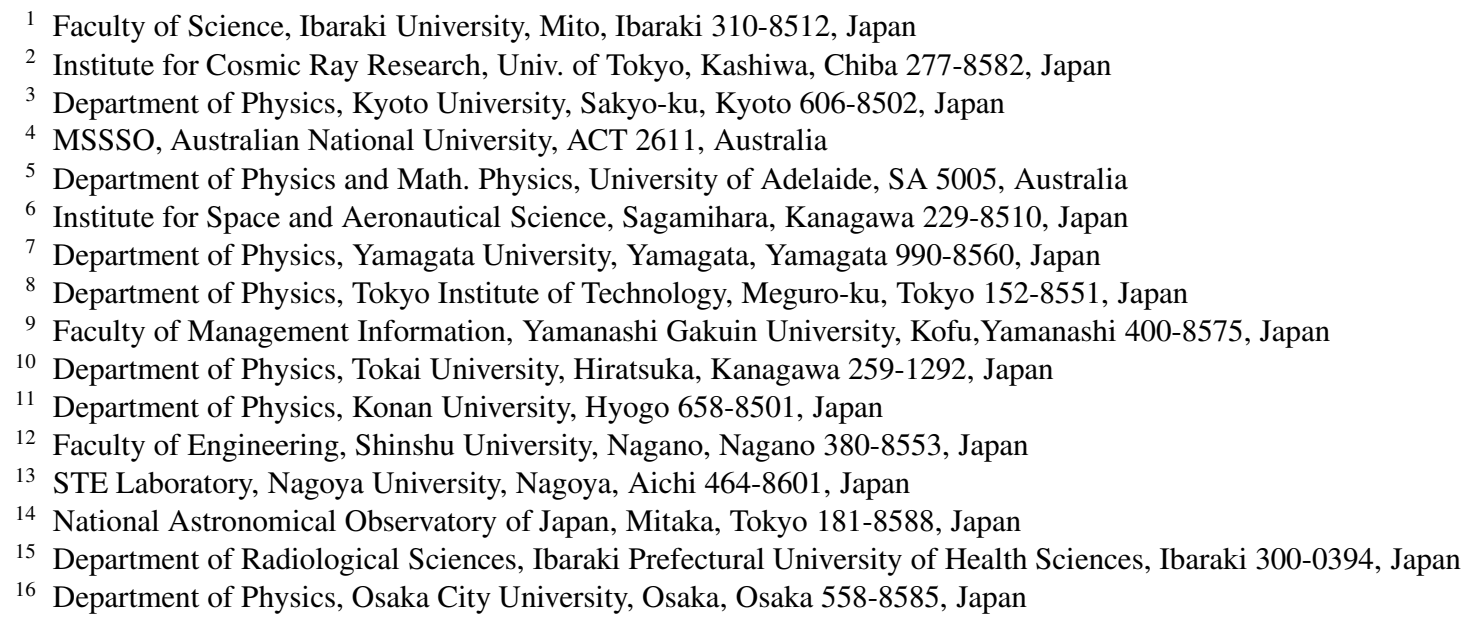

Received 9 September 2002 / Accepted 29 October 2002

\begin{abstract}
We report the TeV gamma-ray observations of the nearby normal spiral galaxy NGC 253. At a distance of $\sim 2.5 \mathrm{Mpc}$, NGC 253 is one of the nearest starburst galaxies. This relative closeness, coupled with the high star formation rate in the galaxy, make it a good candidate $\mathrm{TeV}$ gamma-ray source. Observations were carried out in 2000 and 2001 with the CANGAROO-II $10 \mathrm{~m}$ imaging atmospheric Cerenkov telescope. TeV gamma-ray emission is detected at the $\sim 11 \sigma$ level with a flux of $(7.8 \pm$ $2.5) \times 10^{-12} \mathrm{~cm}^{-2} \mathrm{~s}^{-1}$ at energies $>0.5 \mathrm{TeV}$. The data indicate that the emission region is broader than the point spread function of our telescope.
\end{abstract}

Key words. gamma rays: observation - galaxies: individual: NGC 253 - galaxies: starburst - cosmic rays

\section{Introduction}

NGC 253 is a nearby ( $2.5 \mathrm{Mpc})$ (Vaucouleurs 1978) starburst galaxy, in which a high cosmic-ray density and non-thermal emissions are expected (Voelk et al. 1996). The large-scale radio continuum structure of NGC 253 was studied by Hummel et al. (1984). Carilli et al. (1992) found a large, bright synchrotron-emitting halo extending to more than $10 \mathrm{kpc}$ from the galaxy centre. Diffuse X-ray emission from the halo has also been detected (Cappi et al. 1999; Strickland et al. 2002). The OSSE instrument on the Compton

Send offprint requests to: $\mathrm{R}$. Enomoto, e-mail: enomoto@icrr.u-tokyo.ac.jp
Gamma-Ray Observatory (CGRO) detected sub-MeV gammarays (Bhattacharya et al. 1994) which were attributed to farinfrared photons scattered by the high energy, synchrotron emitting, electrons (Goldshmidt \& Raphaeli 1995). The EGRET detector, however, gave only upper limits on gammaray emission in $\mathrm{GeV}$ energies (Blom et al. 1999).

We have observed NGC 253 with the CANGAROO-II telescope between October 3 and November 18, 2000, and September 20 to November 15, 2001. While the detection of diffuse $\mathrm{TeV}$ emission from our galaxy is very difficult (e.g., Aharonian et al. 2002), observations of NGC 253 provide the opportunity to study the distribution of cosmic rays in galaxies like our own. 


\section{Observation and analysis}

Very high-energy gamma-rays (with energy greater than several hundred $\mathrm{GeV}$ ) interact in the upper atmosphere and produce cascades of secondary photons and particles. Secondary electrons and positrons with energy greater than the (altitude dependent) Cerenkov threshold emit optical photons in directions approximately the same as that of the incident gammaray. The CANGAROO-II telescope (Kawachi et al. 2001) was constructed near Woomera, South Australia $\left(136^{\circ} 47^{\prime} \mathrm{E}\right.$, $31^{\circ} 04^{\prime} \mathrm{S}$ ) to detect the several nano-second duration flashes of Cerenkov light. The telescope consists of a $10 \mathrm{~m}$ reflector and a camera of 552 pixels, each a $0.115^{\circ}$ square photomultiplier (PMT). From the detected Cerenkov light images, the directions and energies of the incident gamma-rays can be reconstructed.

A total of $\sim 75$ hours of observations of NGC 253 ("ON") were made, with a similar amount of background ("OFF") observations. Each $\mathrm{ON}$-source run tracked the center of NGC 253 through its highest elevations, with OFF-source observations offset in right ascension made in order to estimate the background. An edge-on galaxy, NGC 253 appears optically (based on red Digitized Sky Survey [DSS2] data; http: //skyview.gsfc.nasa.gov/), as a disc $\sim 0.4^{\circ}$ by $\sim 0.1^{\circ}$ in size. This is larger than our angular resolution, but much smaller than the telescope's field of view (FOV) of $1.84^{\circ} \times 1.84^{\circ}$. After selecting data taken at high elevation angles $\left(>70^{\circ}\right)$ in good weather conditions, a total of $2959 \mathrm{~min}$. $\mathrm{ON}$-source and $2417 \mathrm{~min}$. OFF-source data remained for further analysis.

First, "cleaning" cuts were applied to the camera images, requiring pixel pulse-heights of greater than $\sim 3.3$ photoelectrons, Cerenkov photon arrival times within $40 \mathrm{~ns}$, and clusters of at least four adjacent triggered pixels in each event. After these pre-selections, we carried out a shower image analysis using the standard set of image parameters, distance, length, width, and $\alpha$ (Hillas 1985), combining length and width (after an initial distance cut) to assign likelihoods to each event (Enomoto et al. 2002a, 2002b). The likelihood for both a gamma-ray origin and cosmic-ray proton origin were calculated. The cut to reject background events was based on the ratio of these two likelihoods. After these cuts, the image orientation angle $(\alpha)$ was plotted. A gamma-ray signal appears as an excess at low $\alpha$ after the normalized OFF-source $\alpha$ distribution is subtracted from the ON-source distribution. As shown in Fig. 1a, an excess of events with $\alpha<30^{\circ}$ is clearly observed for NGC 253 . The number of gamma-ray-like events is $1652 \pm 149(11.1 \sigma)$, corresponding to 0.56 gamma-rays per $\min$.

The energy scale (the conversion factor from ADC value to number of photoelectrons) was determined by adjusting the cosmic ray event rate and the relation between the total $\mathrm{ADC}$ counts and the total number of hit pixels. This estimation also agreed with the calculated single trigger rate for pixel due to the Night Sky Background (NSB). We obtained the threshold energy for the gamma-ray detection to be $\sim 500 \mathrm{GeV}$ for a $E^{-2.5}$ spectrum and $\sim 400 \mathrm{GeV}$ for a $E^{-3.0}$ spectrum, after preselection cuts and also the same values after image parameter

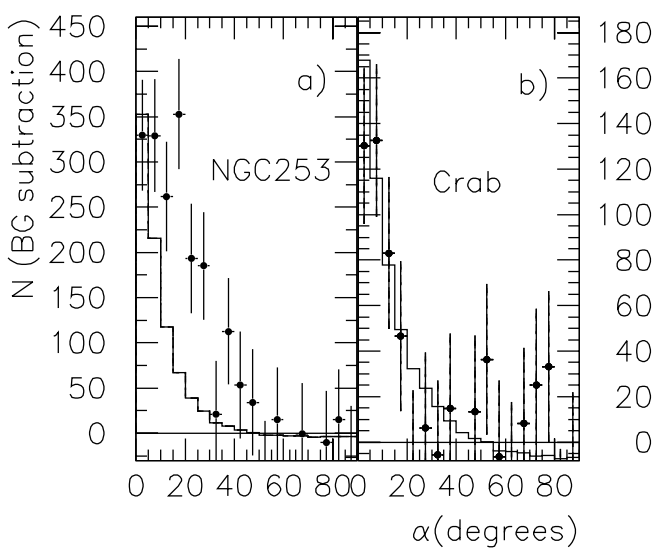

Fig. 1. Distributions of the image orientation angle $(\alpha)$ : a) for NGC 253 and b) for the Crab. The points with error bars were obtained by subtracting the normalized off-source data from the onsource data. The ratio of events in the higher $\alpha\left(>30^{\circ}\right)$ regions for on- and off-source data was used as the normalization factor, which agreed with the ratio of observation times to within $2 \%$. The histograms were obtained from Monte Carlo simulations of gamma-rays from a point-source.

cut. These values were obtained from the peak positions of the effective area multiplied by power law energy dependences.

The same analysis procedure was applied to Crab nebula data from observations in November and December 2000 as a check. Approximately 10 hours of good data were obtained. The resulting $\alpha$ plot, shown in Fig. 1b, has an excess of $393 \pm 59$ events $(6.7 \sigma)$. The solid histograms are the Monte Carlo predictions for the $\alpha$ distribution in the case of a point source. Note that the Crab observation was carried out at large zenith angles, around $56^{\circ}$. At such large zenith angles, the $\alpha$ distribution deteriorates due to the shrinkage of Cerenkov images. In order to compensate for this, we applied tighter cuts in the Crab analysis. As a result, the $\alpha$ distribution for Crab is slightly wider than that for NGC 253 (which is observed near the zenith). The experimental result, however, agrees with the Monte Carlo prediction in case of the Crab observation. For NGC 253, the $\alpha$ distribution is broader than the point spread function (PSF) by a factor of two. Our Monte Carlo simulations predicted that $73 \%$ of events from a point source would have $\alpha<15^{\circ}$, whereas in fact $56 \%$ of events were contained within this range.

\section{Results}

The spatial distribution of the gamma-rays from NGC 253 was studied using the so-called significance map. The thick solid contours in Fig. 2 are our results. The $65 \%$ confidence level contour, which is roughly one standard deviation of our angular resolution, is shown and compared with an optical image from DSS2, which is shown by the solid thin contours. The size of the $\mathrm{TeV}$ emission region is of the same order as the optical image, or larger. The dotted contour is the $65 \%$-contour obtained by the Crab observation. It is consistent with the Monte Carlo prediction of $0.25^{\circ}$. That of the near-zenith observations was estimated to be $0.23^{\circ}$. In order to estimate the telescope 


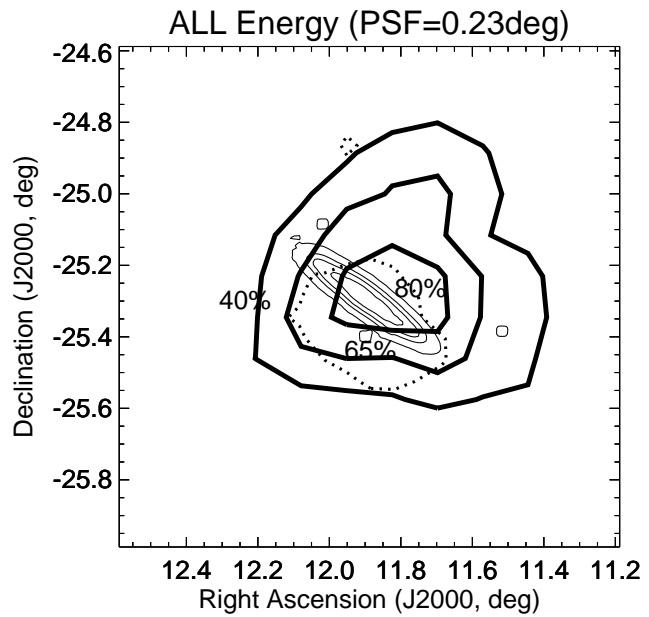

Fig. 2. Profile of the emission around NGC 253. The solid thick contours were obtained from our observations. This was made from the distribution of the detection significance determined at each location from the differences in the $\alpha$ plots (ON- minus OFF-source histogram) divided by the statistical errors. The solid thin contours are the DSS2 (red) data. The dotted contour was obtained with the CANGAROO-II telescope for the Crab.

pointing accuracy, we analyzed bright stars (optical magnitude from 5 to 4 ) in other observations at various elevation angles, as the stars in the NGC 253 field of view are fainter than our detection limit. We conclude that the pointing uncertainty was within $0.1^{\circ}$, which is less than the pixel size. The center of gamma-ray emission from the Crab observation agreed within this accuracy. The gamma-ray acceptance of the telescope gradually decreases beyond $0.3^{\circ}$ from the center of the field of view (dropping to half at $0.8^{\circ}$ ), and so we are unable to make a more detailed morphological study. It is clear, however, that the observed gamma-rays distribution from NGC 253 is inconsistent with emission from a point source.

In order to derive the flux level, we estimated the acceptances (effective areas) using Monte Carlo simulations (Enomoto et al. 2002a). We compared our effective area with that of Whipple (Fig. 6 in Mohanty et al. 1998), which are these after clustering and distance cut, i.e., before image parameter cut. Our effective area agreed with Whipple including the energy dependences. According to Fegan (1996), the threshold of Whipple telescope was the same as ours. The sensitivity for the CANGAROO telescope was represented by that for Crab-type source, i.e., a point source with Crab intensity at zenith. We calculated its acceptance and assumed the same background level, with the cut of $\alpha<15$ degrees. It corresponds to $\sim 8 \sigma$ detection per hour, slightly higher than that of Whipple (Reynolds et al. 1993). A difference appeared after the shape parameter cut, i.e., likelihood analysis. Our acceptance for the likelihood cut was as high as $86 \%$. On the other hands, the "supercut" reduces it to $50 \%$ level (Fegan 1996: Table II). The both background levels are the same. Note that our background level depends on NSB, artificial light background and etc., because we used the experimental OFF sample as the background sample for Probability Density Functions.

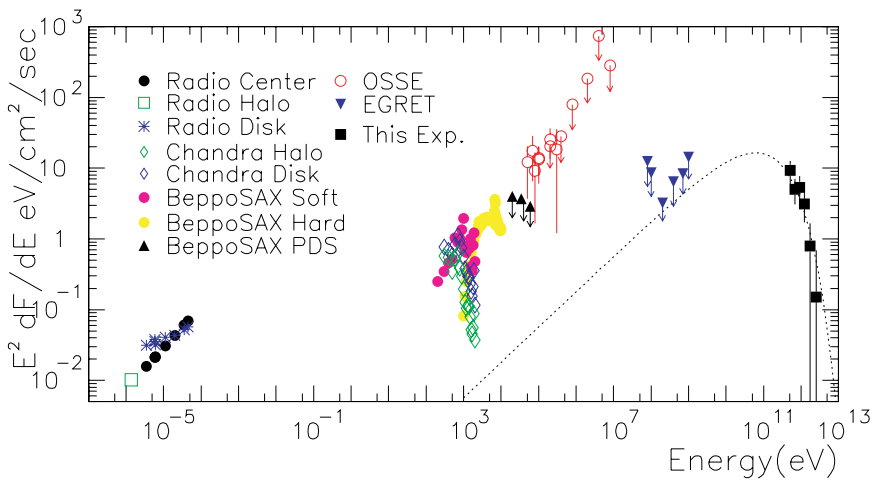

Fig. 3. Multi-band spectrum of NGC 253. The black squares were obtained by this experiment. When we constrain the flux to $3 / 4$ of the EGRET upper limit (Blom et al. 1999) at $0.2 \mathrm{GeV}$ using the function in the text, a flux proportional to $E^{-1.5} \mathrm{e}^{-\sqrt{E} /(0.25 \pm 0.01)}$ was obtained with $\chi^{2} / \mathrm{DOF}=1.8 / 5$ (the dotted line). The $\mathrm{X}$-ray data for CHANDRA and BeppoSAX soft component were corrected for photo-absorption in the Galaxy, but no correction was applied to the BeppoSAX hard X-ray data. No correction was made for photo-absorption inside NGC 253 itself. We note that the BeppoSAX, OSSE, and EGRET data were not able to spatially resolve NGC 253, in contrast to the other data.

The systematic uncertainty was calculated on a bin-by-bin basis by changing the cut values on the likelihood from 0.1 to 0.6 in steps of 0.1 . The uncertainties are typically $\sim 30 \%$. The energy scale uncertainties due to the mirror reflectivity, mirror segment distortions, and Mie scatterings were estimated to be $15 \%$ (point to point) and $20 \%$ (overall). The energy resolution was estimated to be $35 \%$ on an event by event basis. The energy resolution is bad because we could not assume source point event by event basis, i.e., we could not correct it using core distance. The spill over effect, therefore, is considered to be large for such soft energy spectrum. In order to derive the spectrum of NGC 253 self-consistently, we adopted the iteration method. At first we used an acceptance calculated by the simulation with a $E^{-2.5}$ spectrum and we obtained the index of $-3.7 \pm 0.3$. We then iteratively used this fitted value in the simulations to re-derive the spectrum. This process rapidly converged at an index of $-3.75 \pm 0.27$. The same iteration procedure was carried out using a function with cutoff. It also showed a good convergence. The differential fluxes obtained in 2000 and 2001 agreed within these errors. A check was made using Crab nebula data (with an energy threshold of $\sim 2 \mathrm{TeV}$ ) and the derived flux agreed with previous measurements within the statistical errors ( 20\%) (Tanimori et al. 1998; Aharonian et al. 2000). Also, the cosmic ray flux calculated from the background event rate was checked and found to match the expected rate within $10 \%$. A more detailed description of the analysis will be presented elsewhere (Itoh et al. 2002a). The differential fluxes are plotted in the spectral energy distribution (SED) shown in Fig. 3, together with measurements from other energy bands (Carilli et al. 1992; Hummel et al. 1984; Cappi et al. 1999; Strickland et al. 2002; Bhattacharya et al. 1994; Blom et al. 1999).

A simple power-law fit to the TeV spectrum of NGC 253 deviates greatly from the EGRET measurements at GeV energies (Blom et al. 1999): a turn-over below the $\mathrm{TeV}$ region 
clearly exists. We fitted a power law with an exponential cut off, fixing the power-law index to -1.5 , i.e., an inverse Comptonlike spectrum (where the exponential term is $\exp (-\sqrt{E} / a)$, with $a$ a free parameter). The resulting improved fits are shown by the dotted curve in Fig. 3. The minimum power-law index providing an acceptable fit (based on the $\chi^{2}$ value) was -1.8 ( $2 \sigma$ limit). If the observed gamma-rays are produced by inverse Compton scattering of cosmic microwave background (CMB) photons, then the fitted spectrum suggests that the maximum energy of the parent electrons is around several TeV. The integral flux is estimated to be $(7.8 \pm 2.5) \times 10^{-12} \mathrm{~cm}^{-2} \mathrm{~s}^{-1}$ at energies greater than $0.52 \mathrm{TeV}$.

\section{Discussions}

The observations reported here are the first detection of $\mathrm{TeV}$ gamma-rays from a normal spiral galaxy (other than our own), and reveal the emission to be spatially extended and temporally steady. NGC 253 has been observed over a range of photon energies, as depicted in Fig. 3, however the SED is more difficult to interpret. The emission of $50-200 \mathrm{keV}$ photons observed by OSSE (Bhattacharya et al. 1994) was interpreted as being inverse Compton scattering of far-infrared photons from dust in the central region (Goldshmidt \& Raphaeli 1995). A simple extrapolation to higher energies exceeds the EGRET $2 \sigma$ upper limit (Blom et al. 1999). Clearly, it is not possible to explain the OSSE and CANGAROO-II observations and the EGRET upper limits by invoking inverse Compton emission from a single population of electrons. The very large radio halo, extending over $10 \mathrm{kpc}$ (Hummel et al. 1984; Carilli et al. 1992) and up to X-ray energies (Cappi et al. 1999), suggests the existence of a population of very high-energy cosmic rays which may be responsible for the inverse Compton production of $\mathrm{TeV}$ gammarays (Itoh et al. 2002b), quite separate from sources concentrated near the centre of the galaxy which emit the majority of the photons observed by OSSE.

NGC 253 is the first of a new class of object to be detected at $\mathrm{TeV}$ energies. The extended and steady nature of the emission makes it clear that the $\mathrm{TeV}$ gamma-rays are produced in a different environment than that of previously reported extragalactic sources of the active galactic nucleus class.
Studies of NGC 253 will, like those of the LMC at GeV energies (Sreekumar et al. 1992), provide the opportunity to learn more about the cosmic ray environment in galaxies like our own.

Acknowledgements. We thank Prof. T. G. Tsuru for suggestions.

\section{References}

Aharonian, F. A., Akhperjanian, A. G., Barrio, J. A., et al. 2000, ApJ, 539,317

Aharonian, F. A., Akhperjanian, A. G., Barrio, J. A., et al. 2002, Astropart. Phys., 17, 459

Bhattacharya, D., The, L.-S., Kurfess, J. D., et al. 1994, ApJ, 437, 173

Blom, J. J., Paglione, T. A. D, \& Carramiñana, A. 1999, ApJ, 516, 744

Carilli, C. L., Holdaway, M. A., Ho, P. T. P., \& de Pree, C. G. 1992, ApJ, 399, L59

Cappi, M., Persic, M., Bassani, L., et al. 1999, A\&A, 350, 777

Enomoto, R., Hara, S., Asahara, A., et al. 2002a, Astropart. Phys., 16, 235

Enomoto, R., Tanimori, T., Naito, T., et al. 2002b, Nature, 416, 823

Fegan, D. J. 1996, Space Sci. Rev., 75, 137

Goldshmidt, O., \& Rephaeli, Y. 1995, ApJ, 444, 113

Hillas, A. M. 1985, Proc. 19th ICRC (La Jolla), 3, 445

Hummel, E., Smith, P., \& van der Hulst, J. M. 1984, A\&A, 137, 138

Itoh, C. Enomoto, R., Yanagita, S., et al. 2002a, in preparation

Itoh, C., Enomoto, R., Yanagita, Y., Yoshida, T., \& Tsuru, T. G. 2002b, in preparation

Kawachi, A., Hayami, Y., Jimbo, J., et al. 2001, Astropart. Phys., 14, 261

Mohanty, G., Biller, S., Carter-Lewis, D. A., et al. 1998, Astropart. Phys., 9, 15

Reynolds, P. T., Akerlof, C. W., Cawley, M. F., et al. 1993, ApJ, 404, 206

Sreekumar, P., Bertsch, D. L., Dingus, B. L., et al. 1992, ApJ, 400, L67

Strickland, D. K., Heckman, T. M., Weaver, K. A., Hoopes, C. G., \& Dahlem, M. 2002, ApJ, 568, 689

Tanimori, T., Sakurazawa, K., Dazeley, S. A., et al. 1998, ApJ, 492, L33

Vaucouleurs, G. de 1978, ApJ, 224, 710

Voelk, H. J., Aharonian, F. A., \& Breitschwerdt, D. 1996, Space Sci. Rev., 75, 279 